\title{
Quasielastic neutron scattering experiment on water using TOFLAR (Time Of Flight and LARmor precession) technique at SNS
}

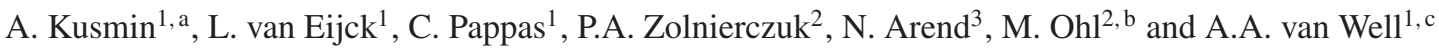 \\ ${ }^{1}$ Department of Radiation Science and Technology, Faculty of Applied Sciences, Delft University of Technology, \\ 2629JB Delft, The Netherlands \\ 2 Jülich Centre for Neutron Science JCNS, Forschungszentrum Jülich GmbH, Outstation at SNS, 1 Bethel Valley Road, \\ Oak Ridge, TN 37831, USA \\ ${ }^{3}$ Jülich Centre for Neutron Science JCNS, Forschungszentrum Jülich GmbH, Outstation at MLZ, Lichtenbergstrae 1, \\ 85747 Garching, Germany
}

\begin{abstract}
We present the results of the first TOFLAR (Time Of Flight and LARmor precession) experiment with a Larmor modulation device positioned after the sample. Using a setup based on the configuration of the SNS-NSE spectrometer (Spallation Neutron Source, USA), we did TOFLAR measurements on water at 5 and 20 degree Celsius, analyzed the spectra, and obtained diffusion coefficient values that are in a reasonable agreement with literature. Thus, our results demonstrate that TOFLAR may become a standard technique to study quasielastic neutron scattering in future.
\end{abstract}

\section{Introduction}

In an inelastic neutron scattering experiment two wavelengths, before and after scattering, have to be determined. Such an experiment can be done using the time-of-flight (TOF) method in two different modes. In a "direct geometry" mode a pulsed incident beam has a fixed incoming wavelength $\left(\lambda_{1}\right)$ and TOF is used to measure the final wavelength $\left(\lambda_{2}\right)$. In an "inverted geometry" mode $\lambda_{2}$ is fixed while TOF is used to measure $\lambda_{1}$. For both modes, only a narrow wavelength band of neutrons (for incoming or scattered neutrons, respectively) is used, resulting in a reduction of available neutron intensity by at least two orders of magnitude, and in limitations for the range in momentum transfers $Q$ and energy transfers $\omega$.

In the TOFLAR technique [1] neither the incoming nor the scattered beam is required to be monochromatic. Larmor precession of polarized neutrons labels $\lambda_{1}$ or $\lambda_{2}$ (depending on whether the Larmor modulator is placed before or after the sample), and TOF yields a combination of $\lambda_{1}$ and $\lambda_{2}$. A schematic setup is shown in Fig. 1.

The results of the first test of the TOFLAR technique were difficult to analyze mainly because of a poor counting statistics [2]. In this paper we present the results of a TOFLAR experiment on water done at SNS with a setup based on the configuration of the SNS-NSE spectrometer.

\section{The TOFLAR technique}

When a Larmor modulator (LM) device is placed after the sample, measured intensity is a function of scattering angle $(\theta)$, TOF channel $\left(t_{C H}\right)$, and the field line integral $(B l)$

\footnotetext{
a e-mail: kusmin@tudelft.nl

b e-mail: m.ohlefz-juelich.de

c e-mail: a.a.vanwell@tudelft.nl
}

Figure 1. A schematic TOFLAR setup. The Larmor modulator (LM) consists of polarizer (P), precession field (B), and analyzer (A), and it can be put either before or after the sample (S).

produced by the LM device:

$$
\begin{aligned}
I\left(t_{C H}, B l\right)= & I_{0} \int_{\lambda_{1}^{\min }}^{\lambda_{1}^{\max }}(1 / 2) p\left(\lambda_{1}\right)\left[1+P_{N S E}\left(\lambda_{2}, B l\right)\right. \\
& \left.\times R\left(\lambda_{2}, B l\right) \cos \left(c \lambda_{2} B l\right)\right]\left(k_{2} / k_{1}\right) S(Q, \omega) \mathrm{d} \lambda_{1}
\end{aligned}
$$

where $\lambda_{1}^{\min } \leq \lambda_{1} \leq \lambda_{1}^{\max }$ is the interval of incident wavelengths, $I_{0}$ is proportional to the integrated neutron flux, $p\left(\lambda_{1}\right)$ is the relative intensity of particular $\lambda_{1}, P_{N S E}$ is the polarization of the sample scattering, $R$ is the resolution function (which accounts for any depolarization not related to the sample scattering), $c=m \gamma / h$ ( $m$ and $\gamma$ are neutron mass and gyromagnetic ratio), $k=2 \pi / \lambda$, $S(Q, \omega)$ is the scattering function of the sample, and $Q=(4 \pi / \lambda) \sin (\theta / 2)$. For simplicity, $\theta$ is assumed to be constant.

Using equations $t_{C H}=(m / h)\left(L_{1} \lambda_{1}+L_{2} \lambda_{2}\right)$ and $\hbar \omega=\left(h^{2} / 2 m\right)\left(1 / \lambda_{2}^{2}-1 / \lambda_{1}^{2}\right)$, and the quasielastic approximation $\left(\lambda_{1} \approx \lambda_{2} \approx \lambda=h t_{C H} /\left(m\left(L_{1}+L_{2}\right)\right)\right)$, Eq. (1) can be written as (more details will be published elsewhere [4]):

$$
\begin{aligned}
I(\lambda, B l)= & \left(I_{0} p(\lambda) / 2\right) \int_{-\infty}^{\infty}\left[1+P_{N S E}(\lambda, B l)\right. \\
& \left.\times R(\lambda, B l) \cos (c \lambda B l) \cos \left(\omega \tau_{T}\right)\right] S(Q, \omega) \mathrm{d} \omega
\end{aligned}
$$

This is an Open Access article distributed under the terms of the Creative Commons Attribution License 4.0, which permits unrestricted use, distribution, and reproduction in any medium, provided the original work is properly cited. 

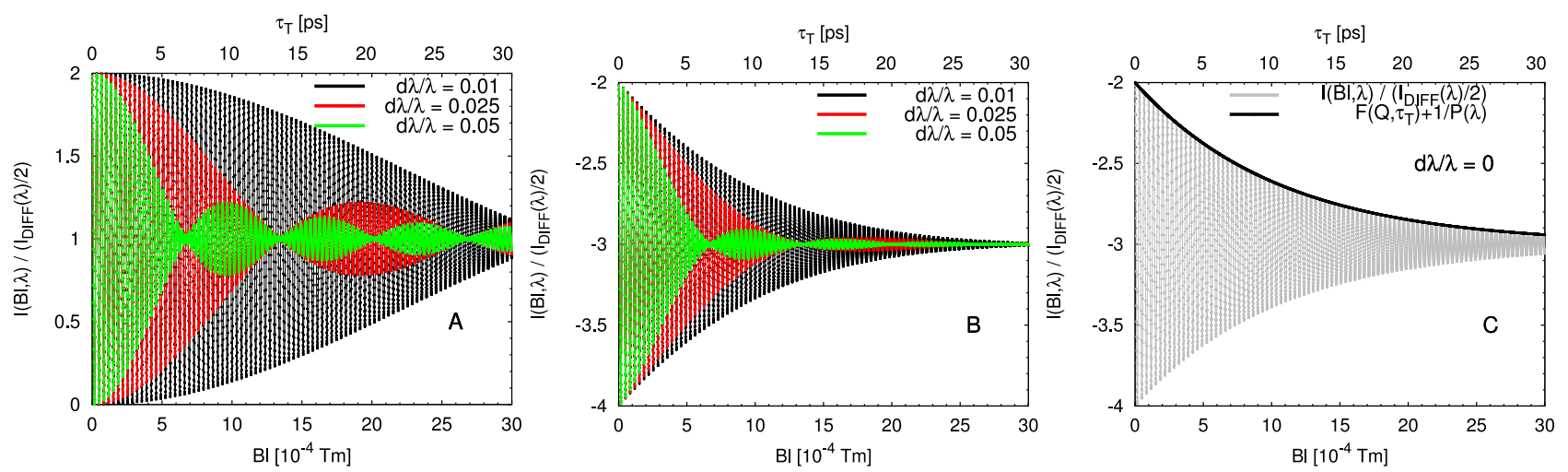

Figure 2. TOFLAR signal calculated for $\lambda=4 \AA$ from Eq. (3) and normalized using Eq. (5a) for the resolution $(\mathrm{A}, P(\lambda)=$ $P_{N S E}(\lambda, B l)=1$ ), and water (B and $\left.\mathrm{C}, P(\lambda)=P_{N S E}(\lambda, B l)=-1 / 3\right) ; R(\lambda, B l)=1$. The envelope of the TOFLAR signal for $d \lambda / \lambda=0$ is the normalized intermediate scattering function $F\left(Q, \tau_{T}\right)$. TOFLAR time $\tau_{T}$ was calculated using $L_{1}$ and $L_{2}$ given in Sect. 3. Calculations for water were done with $F\left(Q, \tau_{T}\right)=\exp \left(-D_{T R} Q^{2} \tau_{T}\right)$, where the translational diffusion coefficient of water is $D_{T R}=90.32 \exp \left(-(573.287 / T)^{2}\right)\left[10^{-5} \mathrm{~cm}^{2} / \mathrm{s}\right]$ [3], and $T$ is the temperature [K]; we used $T=293.15 \mathrm{~K}$.

and TOFLAR time is ${ }^{1} \tau_{T}=\frac{m^{2} \gamma}{2 \pi h^{2}} \frac{L_{1}}{L_{1}+L_{2}} B l \lambda^{3}$. Finally:

$$
\begin{aligned}
I(\lambda, B l)= & \frac{I_{0} p(\lambda) S(Q)}{2}\left[1+F\left(Q, \tau_{T}\right) P_{N S E}(\lambda, B l)\right. \\
& \times(R \lambda, B l) \cos (c \lambda B l)]
\end{aligned}
$$

where $F\left(Q, \tau_{T}\right)$ is the normalized intermediate scattering function, $F\left(Q, \tau_{T}\right)=S\left(Q, \tau_{T}\right) / S(Q)$, where $S\left(Q, \tau_{T}\right)$ is Fourier transform of $S(Q, \omega)$, and $S(Q)$ is the integral of $S(Q, \omega)$ over $\omega$.

An experiment consists of a scan of the field line integral $\mathrm{Bl}$ and yields for each TOF channel a modulated signal such as shown in Fig. 2, where an effect of a finite wavelength resolution (for rectangular resolution function) can be seen as well.

The aim of a quasielastic scattering experiment is to extract $F(Q, t)$ and to test various dynamical models against it. Raw data (described by Eq. (3)) contain a few variables that can be removed by a suitable normalization. Just like in conventional NSE, $I^{+}-$and $I^{-}-$intensities (measured with $\pi / 2$ flippers turned off, and $\pi$-flipper being off, and on, respectively) can be used for this purpose. Using scattered beam polarization $P(\lambda)=$ $\frac{I^{+}(\lambda)-I^{-}(\lambda)}{I^{+}(\lambda)+I^{-}(\lambda)}$, and differential scattering cross-section $S(Q)$, we define $I_{D I F F}(\lambda)=I^{+}(\lambda)-I^{-}(\lambda)$ and $I_{S U M}(\lambda)=$ $I^{+}(\lambda)+I^{-}(\lambda)$ which are

$$
I_{D I F F}(\lambda)=I_{0} p(\lambda) S(Q) P(\lambda) f \approx I_{0} p(\lambda) P(\lambda) S(Q)
$$

$$
I_{S U M}(\lambda)=I_{0} p(\lambda) S(Q)\left(1+P_{P} P_{A}(1-f)\right) \approx I_{0} p(\lambda) S(Q)
$$

where the last approximate identity can be a reasonable approximation (e.g. for small scattering angles/well

\footnotetext{
${ }^{1}$ By contrast to [2], here $L_{1}$, and not $L_{2}$ stands in the numerator because the LM device is after the sample.
}

collimated incident beam, when $\pi$-flipper efficiency $(f)$ is $\geq 0.98) . P_{P}$ and $P_{A}$ are polarizer and analyzer efficiency. Then, two possible normalizations are

$$
\begin{aligned}
& \frac{I(\lambda, B l)}{I_{D I F F}(\lambda) / 2}= \\
& \frac{1+F\left(Q, \tau_{T}\right) P_{N S E}(\lambda, B l) R(\lambda, B l) \cos (c \lambda B l)}{P(\lambda)} \\
& \frac{I(\lambda, B l)}{I_{S U M}(\lambda) / 2}= \\
& 1+F\left(Q, \tau_{T}\right) P_{N S E}(\lambda, B l) R(\lambda, B l) \cos (c \lambda B l) .
\end{aligned}
$$

To extract $F(Q, t)$ of the sample, $R(\lambda, B l)$ must be obtained in advance in a so-called resolution measurement using a sample that exhibits no dynamics $\left(F\left(Q, \tau_{T}\right)=1\right)$ and has no spin-flip scattering (i.e. $P_{N S E}(\lambda, B l)=1$ ).

If Eq. (5a) is used for the normalization, fitting of the following equation

$$
I(\lambda, B l)=a+b \cos (c \lambda B l)
$$

to a normalized signal from the resolution measurement yields $b_{R E S}=R(\lambda, B l) / P_{R E S}(\lambda) ; P_{R E S}$ accounts for any instrument-related depolarization of scattered beam when $\pi / 2$-flippers are off. By fitting Eq. (6) to the sample signal, $b=\left[R(\lambda, B l) / P_{R E S}(\lambda)\right]\left[P_{N S E}(\lambda, B l) / P_{S}(\lambda)\right] F\left(Q, \tau_{T}\right)$ can be obtained; here $P_{S}$ is polarization of neutrons scattered by the sample (when $\pi / 2$ flippers are off) corrected for the instrument-related depolarization. Then, $b / b_{R E S}=\left[P_{N S E}(\lambda, B l) / P_{S}(\lambda)\right] F\left(Q, \tau_{T}\right)$ which is, if all the sample dynamics is due to spin-flip scattering, or if there is no spin-flip scattering at all, just $F\left(Q, \tau_{T}\right)$ (because in such cases $P_{N S E} \equiv P_{S}$ ).

Fitting of Eq. (6) to the resolution and sample signals normalized using Eq. (5b) yields $b_{R E S}=R(\lambda, B l)$ and $b=R(\lambda, B l) P_{N S E}(\lambda, B l) F\left(Q, \tau_{T}\right)$, respectively. Then, $b / b_{R E S}=P_{N S E}(\lambda, B l) F\left(Q, \tau_{T}\right)$, and, if $P_{N S E}=P_{S}$, 


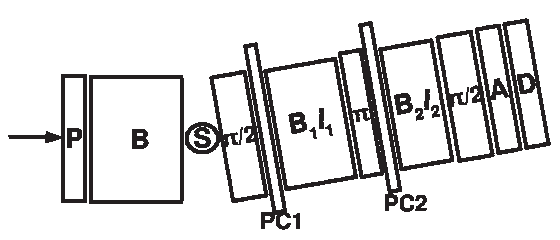

Figure 3. The scheme of the instrument layout. P, B, S, A, and $\mathrm{D}$ stand for polarizer, a guide field to maintain neutron polarization, sample, analyzer, and detector. The first $\pi / 2$-flipper turn the polarization of longitudinally polarized neutrons into the plane perpendicular to the plane of paper, the second $\pi / 2$-flipper does the opposite. Due to the $\pi$ flipper, the net field integral is $B_{1} l_{1}-B_{2} l_{2}$. For a fine adjustment of the net $B l$-integral, either phase coil 1 (PC1) or 2 (PC2) could be used, leading to the net $B l$-integral of $B_{1} l_{1}+B l_{p c 1}-B_{2} l_{2}$ or $B_{1} l_{1}-B_{2} l_{2}-B l_{p c 2}$, respectively.

$F\left(Q, \tau_{T}\right)$ can be readily obtained since $P_{S}$ can be calculated using $I^{+}-$and $I^{-}-$intensities from resolution and sample measurements.

\section{Experimental}

The measurements were done at the SNS-NSE spectrometer at SNS [5,6], modified to have a setup shown in Fig. 3. Distances between the source and the sample, and the sample and detector were $L_{1}=23.361 \mathrm{~m}$ and $L_{2}=$ $3.939 \mathrm{~m}$, respectively. Larmor modulation was set up in the second arm of the spectrometer to have a better TOF resolution (because $L_{1} \gg L_{2}$ ).

The wavelength range, $3.6 \AA \leq \lambda \leq 6 \AA$, was split into 1001 TOF channels. Measurements were done at $\theta=25^{\circ}$ (we tried to measure at $\theta=70^{\circ}$ but the polarization was not sufficiently high). Water was measured at $20^{\circ} \mathrm{C}$ and $5^{\circ} \mathrm{C}$. The sample container was an aluminium rectangular flat cell (one of the standard containers used at BASIS, SNS); we used aluminium spacers to achieve water layer thickness of $0.25 \mathrm{~mm}$. Resolution measurements were done with a zero alloy TiZr instead of Grafoil because of the targeted $Q$-region $Q>0.5 \AA^{-1}$. This alloy has only isotopic incoherent (non-spin flip) scattering which is almost isotropic [7]. We did not measure scattering by either empty cell or empty instrument. Counting times per one $B l$-point were $750 \mathrm{~s}, 900 \mathrm{~s}$, and $600 \mathrm{~s}$ for resolution, and water at $20^{\circ} \mathrm{C}$, and $5^{\circ} \mathrm{C}$, respectively. For analysis, counts over the whole position sensitive detector were summed up; to further improve counting statistics 1001 TOF channels were grouped into 101 channels.

It is obvious from Fig. 2 that to measure TOFLAR signal at closely spaced $B l$-values (to see signal oscillations) in a wide $B l$-interval a very long measurement time is required. Therefore, we selected six narrow $B l$-intervals centered at different $B l=B_{1} l_{1}-B_{2} l_{2}$, and slightly varied the net $B l$ by a fine adjustment of a phase coil current (see Fig. 3) to measure $20 \mathrm{Bl}$-points per interval. We intended to use the phase coil 2 but due to a misconfiguration used the phase coil 1 instead. To take that into account, the net $B l$ values were recalculated by the NSE control program. For every $B l$-interval, $I^{+}$and $I^{-}$were measured at one $B l$ in the center of an interval.

\section{Results}

\subsection{Raw data}

Intensities (in the form of $I_{S U M}(\lambda)=I^{+}(\lambda)+I^{-}(\lambda)$ ) and polarizations of scattered neutrons, flipping ratios, and normalized TOFLAR signals are shown in Fig. 4. As seen from Eq. (4b), if $S(Q)$ is constant within our $Q$-region of $0.45 \AA^{-1} \leq Q \leq 0.75 \AA^{-1}$ (this is true for TiZr and $\left.\mathrm{H}_{2} \mathrm{O}\right)$, then $\bar{I}_{S U M}(\lambda)$ is proportional to $p(\lambda)$. The dips in $p(\lambda)$ come from Bragg reflections from aluminium (for $\lambda \approx 4.05$ and $4.67 \AA$ corresponding to $Q \approx 2.69$ and $3.1 \AA^{-1}$ ) and from Si wafers of the bender $\left(\lambda \approx 5.16 \AA, Q \approx 2.43 \AA^{-1}\right)$. Those dips correspond to the dips in polarization. Note that after correction for the instrument-related beam depolarization, polarization of water scattering is $\approx-0.2 / 0.6=-0.33$ close to theoretical $P=-1 / 3$ for spin-incoherent scattering. This is an indication that multiple scattering and the absence of background corrections have no large effect. The flipping ratio generally increases with increasing $\lambda$ but is lower than expected for small $B l \mathrm{~s}$ such as here. Finally, the TOFLAR signal for a constant $B l$ as a function of $\lambda$ demonstrates oscillations according to $\cos (c \lambda B l)$. For water, oscillations have a smaller amplitude than for the resolution sample (as expected for a smaller (by modulus) polarization), and a phase shift by $180^{\circ}$ (because of spin-incoherent scattering).

Note that the normalized TOFLAR signal does not oscillate around unity (as expected after normalization using Eq. (5b)). This may indicate that the phase coil 1 (actually used for a fine adjustment of the net $B l$-integral) interfered with a function of the first $\pi / 2$ flipper.

The TOFLAR signal shown in Fig. 4 allows an independent determination of $B l$ by fitting $\cos (c \lambda B l)$ to it. When we first tried to do this, we found that fitted $B l$ values were by $\approx 4 \times 10^{-4} \mathrm{Tm}$ larger than the nominal $B l$ s calculated from the magnetic field configuration. Another demonstration that nominal $B l$ should be corrected is given in Fig. 5. We determined $B l$-offset $\left(B l_{0}\right)$ by fitting $\cos (c \lambda B l)$ to all spectra $(I(B l, \lambda))$ measured at $120 B l$ values (six $B l$-intervals, 20 values per an interval), it was almost constant: $B l_{0}=3.9 \pm 0.1 \times 10^{-4} \mathrm{Tm}$. The offset is known to exist (it is due to the flux pinning in superconducting solenoids) and was determined earlier [8] to be $\tau_{N S E}=5.0 \pm 0.9 \mathrm{ps}$ for $4 \leq \lambda \leq 7 \AA$ (which translates to a $B l_{0} \approx 4 \times 10^{-4} \mathrm{Tm}$ ).

TOFLAR signals (unnormalized in order to show counting statistics) are plotted as a function of corrected $B l$-values for every $B l$-interval in Fig. 6 where, again, a $180^{\circ}$ shift for water signal can be seen.

\subsection{QENS analysis of TOFLAR signal from water}

From the above results it is apparent that error bars for water measurements are rather large, and that grouping of a large number of TOF channels together would lead to smaller error bars at the expense of damping the modulated signal due to an increase in $d \lambda / \lambda$ (see Fig. 2). There are two major concerns regarding the use of Eq. (6) to extract $F\left(Q, \tau_{T}\right)$ of water. First, normalizations using Eqs. (5a), (5b) lead to an increase of error bars, especially in case of normalization to $\left(I^{+}-I^{-}\right) / 2$. 

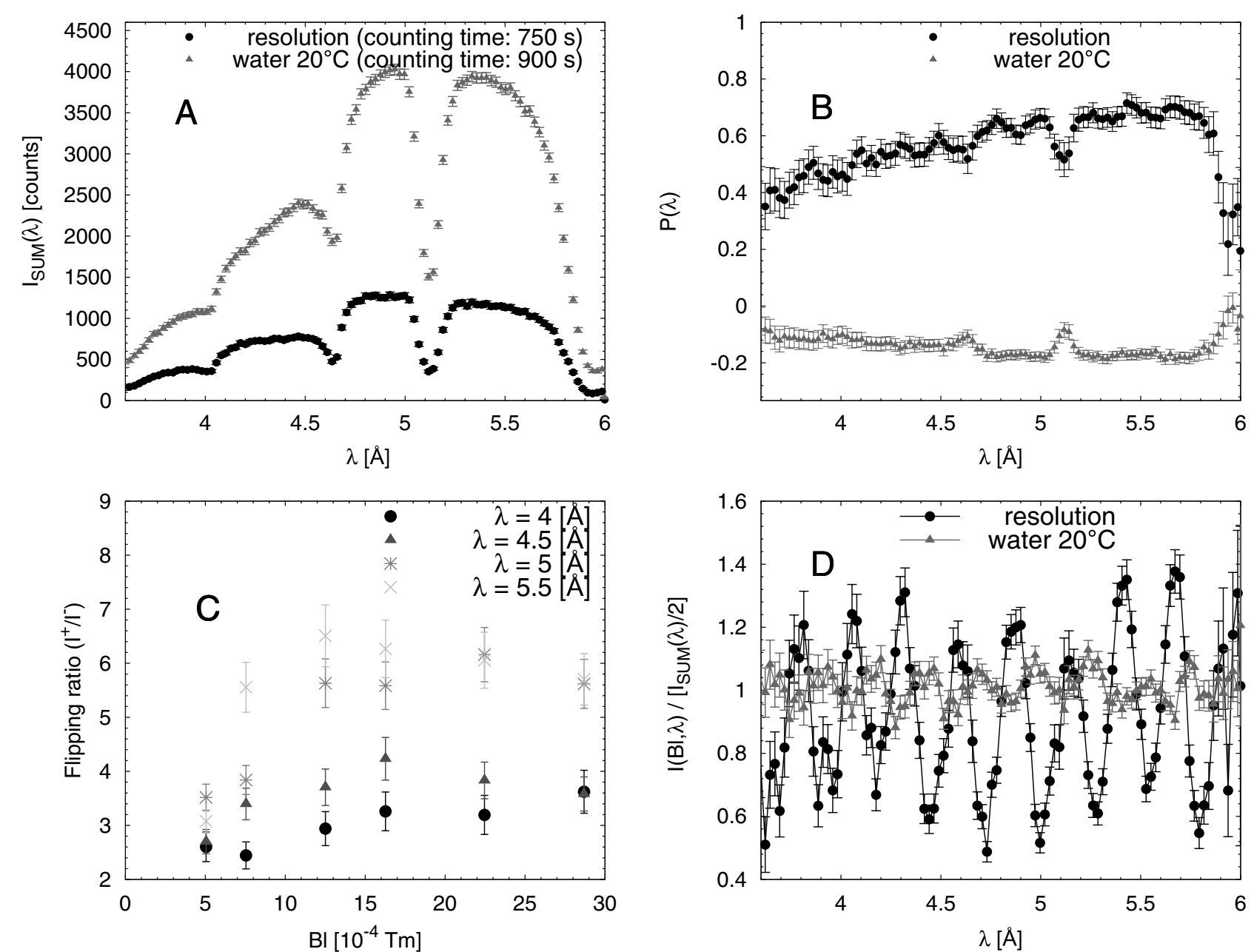

Figure 4. Plot of $I_{S U M}(\lambda)=I^{+}(\lambda)+I^{-}(\lambda)(\mathrm{A}), P(\lambda)=\frac{I^{+}(\lambda)-I^{-}(\lambda)}{I^{+}(\lambda)+I^{-}(\lambda)}$ normalized according to Eq. (5b) (D).

Second, since $\tau_{T}$ is proportional to $B l$, by using Eq. (6) we obtain an average of $F\left(Q, \tau_{T}\right)$ over the $\tau_{T}$-interval that corresponds to a given $B l$-interval. Thus, depending on the $\tau_{T}$-dependence of $F\left(Q, \tau_{T}\right)$, and on the width of the $B l$-interval, extracted $F\left(Q, \tau_{T}\right)$-values may deviate significantly from the true $F\left(Q, \tau_{T}\right)$.

Therefore, we chose to analyze TOFLAR signal that was only normalized to the integrated neutron flux, and we first fitted

$$
I(B l, \lambda)=a_{R E S}+b_{R E S} \cos \left(c \lambda\left(B l+B l_{0}\right)\right)
$$

to the resolution signal (where $F\left(Q, \tau_{T}\right) \equiv 1$ ); free fitting parameters were $a_{R E S}, b_{R E S}$ and $B l_{0}$. Then we fitted to the sample signal the equation

$$
I(B l, \lambda)=a+b b_{R E S} F\left(Q, \tau_{T}\right) \cos \left(c \lambda\left(B l+B l_{0}\right)\right)
$$

where $b_{R E S}$ was fixed, and $a, b$, and $B l_{0}$ were free fitting parameters (we did not fix $B l_{0}$ to respective values obtained from fitting of Eq. (7) to the resolution signal because $B l_{0}$ is generally slightly different in every measurement). The expression for $F\left(Q, \tau_{T}\right)$ contains additional free fitting parameters that depend on the model used to describe water dynamics. To model $F\left(Q, \tau_{T}\right)$ in our $Q$-region long-range translational diffusion coefficient $D_{T R}$, mean residence time $\left(\tau_{0}\right)$ between jumps, and
(B), flipping ratios for the resolution sample $(\mathrm{C})$, and $I(B l, \lambda)$

rotational diffusion coefficient $\left(D_{r}\right)$ are required $[9]^{2}$ but to reliably determine all three parameters a wide $Q$-region extending to larger $Q$-values is needed. Therefore, we used $F\left(Q, \tau_{T}\right)=\exp \left(-D_{T R} Q^{2} \tau_{T}\right)$.

To keep the $Q$-range and $\tau_{T}$-range as wide as possible, TOFLAR signals measured in all TOF channels and in all $B l$-intervals should be used. But, as seen in Fig. 4A, some TOF channels have much smaller intensities than others; we chose to use only channels with particularly high intensities $(4.8 \leq \lambda \leq 5 \AA$ and $5.2 \leq$ $\lambda \leq 5.7 \AA$ ) to decrease uncertainties in fitted parameter values (at the cost of reducing $Q$-region to $0.48 \leq Q \leq$ $0.57 \AA^{-1}$ ). Furthermore, we chose to use only the first two $B l$-intervals because a finite wavelength resolution (due to a pulse width and due to summing of several TOF channels together) destroys the signal at larger $B l$ s, see Figs. 2, 6.

\footnotetext{
${ }^{2}$ For $F(Q, \tau)=\exp \left(-\Gamma_{T R} \tau\right)\left[A_{0}+\sum_{i}^{\infty} A_{i} \exp \left(-\Gamma_{R i} \tau\right)\right] \quad$ [9] and $\quad Q_{M A X}=0.75 \AA^{-1}, \quad \Gamma_{T R}=D_{T R} Q^{2} /\left(1+\tau_{0} D_{T R} Q^{2}\right) \approx$ $D_{T R} Q^{2} / 1.11$ for $\tau_{0}=1 \mathrm{ps}, D_{T R}=2 \times 10^{-5}\left[\mathrm{~cm}^{2} / \mathrm{s}\right]$. Also, $A_{0}=(\sin (Q R) / Q R)^{2}=0.91$ for $R=0.98 \AA$. Thus, the assumption $F(Q, \tau)=\exp \left(-\Gamma_{T R} \tau\right)$ with $\Gamma_{T R}=D_{T R} Q^{2}$ is approximate, but is difficult to assess its validity without exact knowledge of $\tau_{0}$ and $D_{r}$ : setting $\tau_{0}=0$ leads to an underestimated $D_{T R}$, but the neglect of rotational diffusion leads to an overestimated $D_{T R}$.
} 

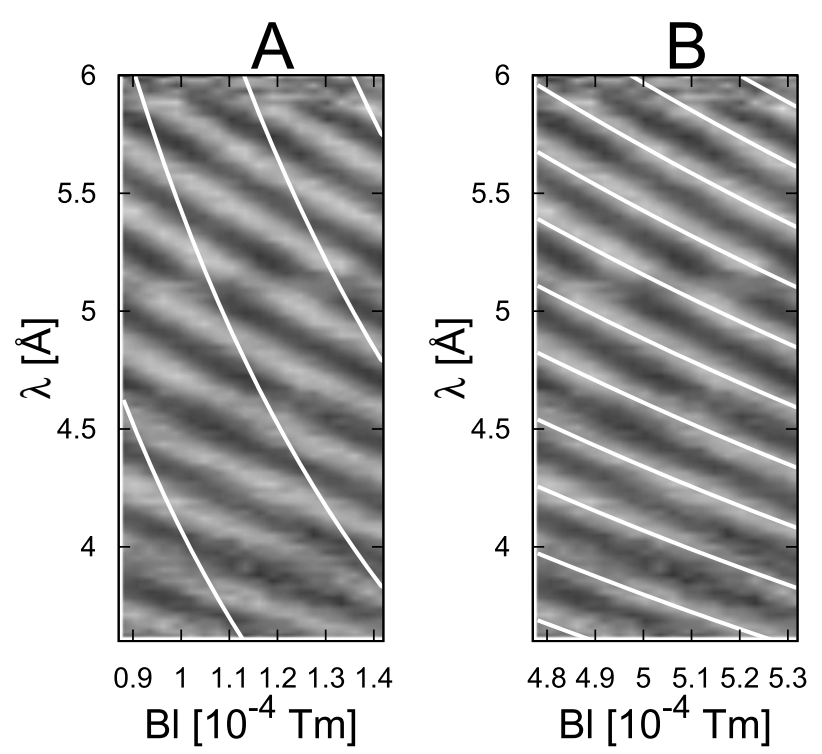

4.84 .955 .15 .25 .3

$\mathrm{BI}\left[10^{-4} \mathrm{Tm}\right]$

Figure 5. Plots of the TOFLAR signal (normalized to $I_{S U M}(\lambda) / 2$ ) using nominal (A) and corrected (by applying an offset of $3.9 \times$ $\left.10^{-4} \mathrm{Tm}\right) B l$-values (B). White lines correspond to the parametric equation $c \lambda B l=2 \pi N$ for $N=1,2$. The slope of white lines must be the same as the slope of grey stripes corresponding to the maximum signal.

At first we fitted Eqs. (7), (8) to TOFLAR signals measured in several $B l$-intervals for one single TOF channel; we did it channel by channel. $a_{R E S}, b_{R E S}, a, b$, and $B l_{0}$ were interval-dependent fit parameters, $D_{T R}$ was an interval-independent fit parameter. Thus, we obtained a range of $D_{T R}(\lambda)$-values, but all those $D_{T R}$ 's had large uncertainties (because of experimental error bars and because the number of fitting parameters per TOF channel (for both resolution and sample) was $N \times 3+1$, where $N$ is the number of fitted $B l$-intervals, and the number of data points was $N \times 20$ ).

To reduce the number of free fitting parameters, we used an extra constraint, namely that $D_{T R}$ is $\lambda$ independent, and simultaneously fitted Eqs. (7), (8) to all TOF channels. $D_{T R}$ was a single global fit parameter, $B l_{0}$ was $B l$-interval-dependent but $\lambda$-independent fit parameter, while $a$ and $b$ were $B l$-interval- and $\lambda$ dependent fit parameters. Thus, when $M$ TOF channels were used, the number of free fitting parameters per one TOF channel was $(2 \times N \times M+M+1) / M \approx 2 \times$ $N+1$. However, even in this approach uncertainties in fitted $D_{T R}$-values were too large. To further decrease them, we made $a$ and $b$ to be $B l$-interval independent fit parameters and obtained $D_{T R}$ of $1.35 \pm 0.3$ and $2.37 \pm$ $0.39\left[10^{-5} \mathrm{~cm}^{2} / \mathrm{s}\right]$ for water at $5^{\circ} \mathrm{C}$ and $20^{\circ} \mathrm{C}$, respectively (literature value are 1.5 and $1.97\left[10^{\circ}-5 \mathrm{~cm}^{2} / \mathrm{s}\right]$ ). The agreement is reasonable taking into account $i$ ) poor counting statistics, $i$ ) an uncertainty due to a $B l$-offset, and iii) a limited $Q$-region where both $\tau_{0}$ and $D_{r}$ must be used in addition to $D_{T R}$ to accurately model the intermediate scattering function of water.

In principle, instead of analyzing TOFLAR data as a set of spectra measured at fixed wavelengths, we could analyze $I(B l, \lambda)$ as a set of spectra measured at fixed $B l \mathrm{~s}$, but then a correction for $p(\lambda)$ would be necessary,
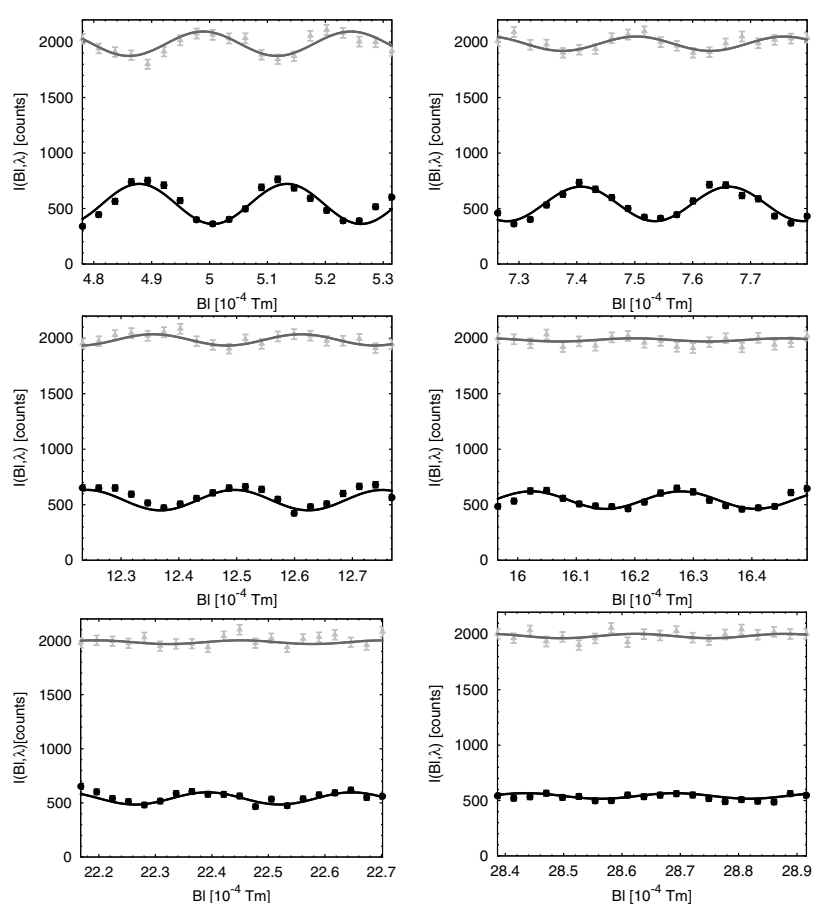

Figure 6. Unnormalized TOFLAR signal (summed over the detector) for the resolution (black, counting time $750 \mathrm{~s}$ ) and water at $20^{\circ}$ (light grey, counting time $900 \mathrm{~s}$ ); $\lambda \approx 5.3 \AA, B l_{0}=3.9 \times$ $10^{-4} \mathrm{Tm}$ was used to correct $B l$-values. Lines are fits to Eq. (6).

and, consequently a normalization (cf. Eqs. (5a), (5b)) would be needed. In any case, to better assess different data reduction and analysis routes, experimental data of better statistical accuracy are required. Such data can in principle be obtained by optimizing the instrument setup to have higher flipping ratios, higher polarization of scattered beam, and/or by choosing a non-spin-flip scattering sample.

The reader might ask: "If TOFLAR brings a large intensity gain due to the absence of monochromatization, why better statistical accuracy could not be achieved for water?" Apart from various reasons already mentioned above, it is also because an important idea of the TOFLAR technique was not implemented, which is to have both polarizer and analyzer either before or after the sample [1]. In our case, by having polarizer after the sample, the polarization of scattered neutron would be $\approx 1$ instead of $-1 / 3$, leading to a significant improvement of measured signals.

\subsection{Conclusion}

We performed the first TOFLAR experiment with a Larmor modulation device positioned after the sample using a slightly modified SNS-NSE spectrometer setup at SNS, USA. After testing various data reduction and data analysis routes we successfully analyzed TOFLAR spectra of water recorded at two temperatures and extracted diffusion coefficients that are in a reasonable agreement with literature values. Thus, the results of our proof-of-principle experiment call for further experiments in order to test practical applicability of TOFLAR technique, and its performance compared to TOF-QENS 
and classical NSE techniques, and in order to develop optimal measurement and data analysis strategies.

We thank Mr. M. Cochran for technical assistance. The neutron scattering experiments at Oak Ridge National Laboratory's (ORNL) Spallation Neutron Source were supported by the Scientific User Facilities Division, Office of Basic Energy Sciences, U.S. Department of Energy (DOE). ORNL is managed by UTBattelle, LLC, for the U.S. DOE under Contract No. DEAC05-00OR22725.

\section{References}

[1] F.M. Mulder, R. Kreuger, S.V. Grigoriev, W.H. Kraan, M.T. Rekveldt, A.A. van Well, Physica B 276, 132 (2000)
[2] M. Bleuel, A.A. van Well, Physica. B 406, 2478 (2011)

[3] T. Dippel, Ph.D. thesis, Max-Planck-Institut (1991)

[4] A.A. van Well, to be published (2014)

[5] M. Ohl, M. Monkenbusch, T. Kozielewski, B. Laatsch, C. Tiemann, D. Richter, Physica. B 356, 234 (2005)

[6] M. Ohl, M. Monkenbusch, N. Arend, T. Kozielewski, G. Vehres, C. Tiemann, M. Butzek, H. Soltner, U. Giesen, R. Achten et al., Nucl. Instrum. Methods Phys. Res. ,. Sect. A. 696, 85 (2012)

[7] S.S. Sidhu, L.R. Heaton, D.D. Zauberis, F.P. Campos, J. Appl. Phys. 27, 1040 (1956)

[8] M. Cochran, T. Kozielewski, M. Ohl, L. Stingaciu, P.A. Zolnierczuk, unpublished (2013)

[9] J. Teixeira, M.C. Bellissent-Funel, S.H. Chen, A.J. Dianoux, Phys. Rev. A. 31, 1913 (1985) 